\title{
The adsorption of protein molecules at a crystal/solution interface observed by an improved TIRFM
}

\author{
Guoliang Dai \\ National Microgravity Lab, Institute of Mechanics \\ Chinese Academy of Sciences \\ Beijing, China \\ dspr@imech.ac.cn
}

\author{
Gen Sazaki \\ The Institute of Low Temperature Science \\ Hokkaido University \\ Hokkaido, Japan \\ sazaki@lowtem.hokudai.ac.jp
}

\begin{abstract}
We have improved the ordinary total internal reflection fluorescence microscopy (TIRFM). Two improvements have been achieved, one is the interface between opaque material and solution can be observed, another is the interface far away (usually several ten micro meters) the objective lens can be observed. By this improved TIRFM, the adsorption of protein molecules at a crystal/solution interface had been successfully observed. We have obtained the results of relationship between the amount of adsorbed protein molecules on bunched steps and the height of bunched steps of a protein crystal.
\end{abstract}

Keywords-TIRFM; adsorption; solid/solution interface

\section{INTRODUCTION}

Total internal reflection fluorescence microscopy (TIRFM) is one of the most promising bio-optics techniques in the world, which have been used to observe fluorescent molecules directly in cells, tissues, polymers, glass, and so on. TIRFM fascinate scientists in fields ranging from biophysics [1-4] to cellular biology [5], to materials and polymer science [6-8]. After the information of individual molecules is extracted from the ensemble observation, scientists can obtain the results after removing ensemble averaging and can compare these statistical results with theoretical models in a variety of cases. However, there are still some disadvantages of ordinary TIRFM. For example, there is a limitation of observation distance (usually around $0.2 \mu \mathrm{m}$ ) between objective lens of TIRFM and object of interest. Moreover, the object of interest has to be transparent; otherwise, the incident laser beam can not penetrate the object of interest and excite the fluorescence group on the label fluorescent molecules. In many scientific fields, such as, material science, the object of interest is usually opaque. In order to change the current situation, we have improved the ordinary TIRFM. By the improved TIRFM, one can not only observe the interface between solid (including almost all solid materials, transparent or opaque) object of interest and solution, but also observe the object of interest a couple of ten micro meters away from the object lens. In this work, we used the improved TIRFM to observe the interface between a model protein (monoclinic hen egg white lysozyme, HEWL) crystal surface and solution in a single molecular level. We obtained the relationship between the amount of adsorbed HEWL molecules on bunched steps and the height of bunched steps of a HEWL crystal.

\section{MATERIALS AND METHOD}

We improved the ordinary TIRFM by adding five mirrors to the optical path for adjusting the angle and movement of the laser beam. (see detailed in Fig. 1). In our improved TIRFM, a high sensitive EM-CCD system (Andor Technology Ltd, United Kingdom) and a specific objective 60X oil immersion lens (PlanApo, Olympus) with large aperture value (N.A.=1.45, W.D. $=0.15 \mathrm{~mm}$ ) were included. The wavelength of laser beam used in the improved TIRFM and LCM-DIM were 532 and $633 \mathrm{~nm}$, respectively.

Commercial HEWL: six times re-crystallized (Seikagaku Kogyo Co., Cat. No. 100940). All other chemicals were of reagent grade. Fluorescence labeled HEWL (F-HEWL): commercial HEWL was chemically modified with tetramethylrhodamin-5-isotiocyanate (5-TRITC, Molecular Probes T-1480) [9]. The number of 5-TRITC groups reacted with a HEWL molecule was examined by sodium dodecylsulfate polyacrylamide gel electrophoresis (SDSPAGE). Only one amino group on the surface of a HEWL molecule was chemically modified with 5-TRITC and the mole ratios of 5-TRITC to the amino groups of HEWL was 1.0. The concentration of F-HEWL in our experiments was $0.1 \mathrm{nM}$. Purified HEWL (purity: 99.99\%): purchased from Maruwa Food Industries, Inc., Japan. Buffer solution: $50 \mathrm{mM}$ sodium acetate solution, $\mathrm{pH}$ 4.5. The final concentration of $\mathrm{NaNO}_{3}$ in HEWL bulk solution was $20 \mathrm{mg} / \mathrm{ml}$. Laser Confocal Microscopy and Differential Interference Microscopy (LCMDIM) system was described in detail in [10]. The temperature inside the observation chamber (shown in Fig. 2) was controlled at $19 \pm 0.1^{\circ} \mathrm{C}$.

G. Dai thanks grants from the National Nature Science Foundation of China (Nos. 20603043, 10432060). 


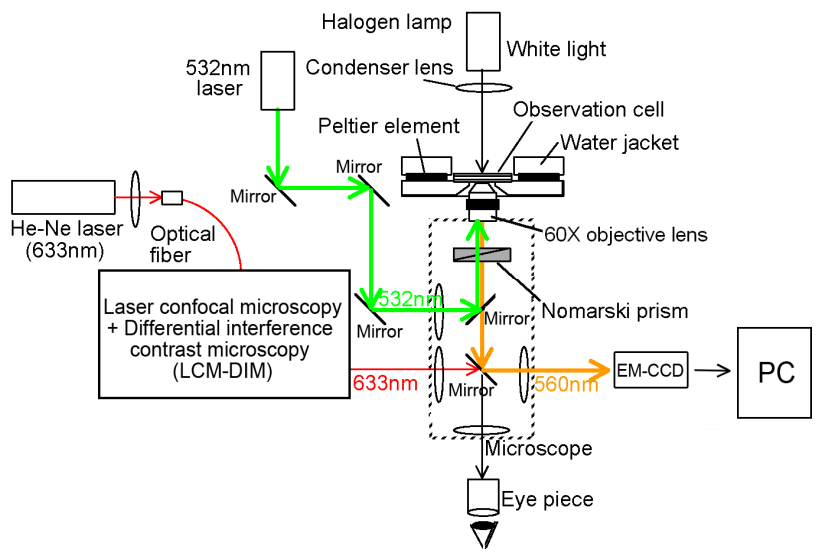

Figure 1. Schematic structure illustration of the improved TIRFM.

\section{RESULTS AND DISCUSSION}

The distance between the object of interest and the objective lens is often several ten micro meters in the improved TIRFM, which is much larger than that of ordinary TIRFM. Thanks to this advantage, both crystal/solution interfaces, including upper and lower interfaces, can be observed (shown in Fig. 2). In case of opaque object of interest, lower interface is observed after adding spacers (with different diameters, usually, $1 \mu \mathrm{m}$ ). In case of transparent object of interest, such as in our experiments, upper interface is observed without any spacer.

After the improvement of TIRFM, we chose an upper crystal/solution interface, on which the surface flatness was bad and had a special shape, to be observed in the same field of view by LCM-DIM and the improved TIRFM. The results shown in Fig. 3 showed that the special shape could be clearly shown, which proved that the upper interface can be observed by the improved TIRFM.

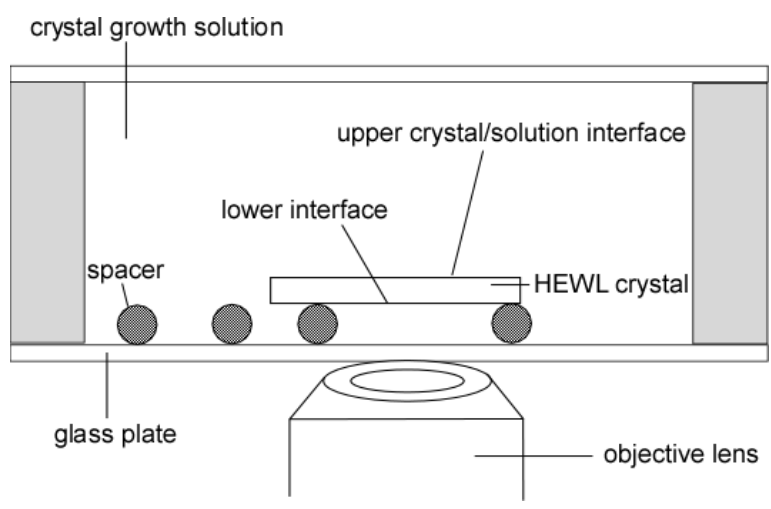

Figure 2. The observation chamber and the crystal/solution interface observed by the improved TIRFM. The distance between the observed interface and the objective lens is much larger than that of ordinary TIRFM

The upper crystal/solution interface was observed in this work.
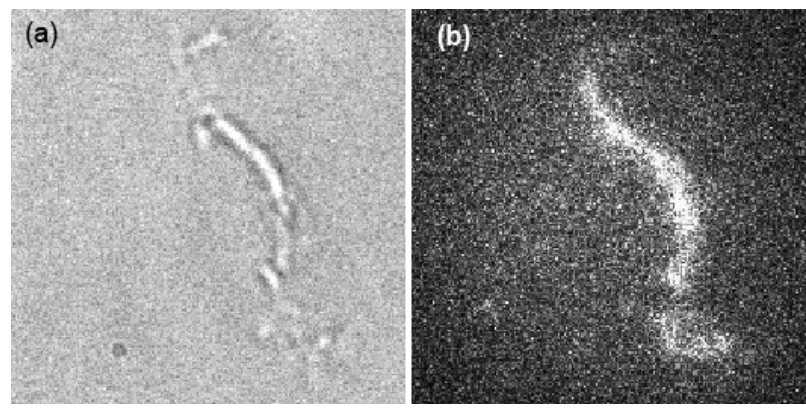

Figure 3. The morphologies of a crystal surface in the same field of view (with a special shape) taken by LCM-DIM (a) and improved TIRFM (b). The scales of (a) and (b) are $17 \mu \mathrm{m} \times 17 \mu \mathrm{m}$.
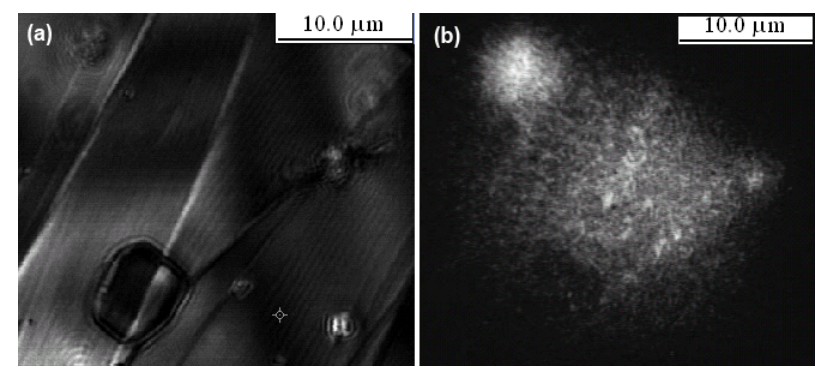

Figure 4. F-HEWL molecules adsorbed on the crystal surface and part of the bunched steps. (a): observed by LCM-DIM. (b): observed by the improved TIRFM in the same field of view.

We observed and counted the F-HEWL molecules on the bunched steps of the HEWL crystal surface $2 \mathrm{~h}$ after F-HEWL solution was introduced into the observation chamber. The images taken by LCM-DIM and the improved TIRFM were shown in Fig.4 (a) and (b), respectively. Both images were taken in the same field of view. Parts of big bunched steps can be observed both in Fig. 4(a) and (b). One bright dot corresponds to one adsorbed F-HEWL molecule on the HEWL crystal surface. Fig. 4(b) indicates that the positions of single F-HEWL molecules on the HEWL crystal surface can be cleared observed. Fig. 4 (b) also showed that the amount of the F-HEWL molecules adsorbed on bunched steps was obviously much larger than that on a terrace. This result was consistent with the description "steps can also be a host for foreign particles that preferentially attach along the step, producing thereby a linear chain of atoms" in [11]. In [12], the impurity molecules, HEWL dimer, tend to adsorb on a terrace than on steps. The difference between their results and ours were caused by the impurity molecules used themselves.

We counted the total number of F-HEWL molecules adsorbed on bunched steps of different height. The result was shown in Fig. 5. The height of bunched steps was measured according to the following equation:

$$
h=\frac{\lambda}{2} \cdot \frac{s}{l}
$$

Where $h$ is the height of the bunched step, $s$ is the shift distance of interference fringes, $\lambda$ is the wavelength of the 
incident laser beam, $l$ is the distance between two vicinal interference fringes. The interference fringe can be observed by LCM-DIM when the bunched step is high enough.

Fig. 5 clearly showed that the amount of adsorbed FHEWL molecules on bunched steps in unit area has a linear relationship with the height of bunched steps as the function of $\mathrm{Y}=-0.00296+0.00119 \mathrm{X}$, where $\mathrm{Y}$ is the amount of adsorbed F-HEWL molecules in unit observation area, $\mathrm{X}$ is the height of the bunched steps.

\section{CONCLUSION}

We have improved the ordinary TIRFM and successfully observed the interface of HEWL crystal/solution. The adsorption behavior of F-HEWL molecules on a single molecular level was consistent with the previous experimental results, i.e. F-HEWL molecules were preferentially adsorbed on bunched steps than on a terrace. By the improved TIRFM, we obtained the relationship between the number of adsorbed F-HEWL molecules on the bunched steps of HEWL crystal surface and the height of bunched steps.

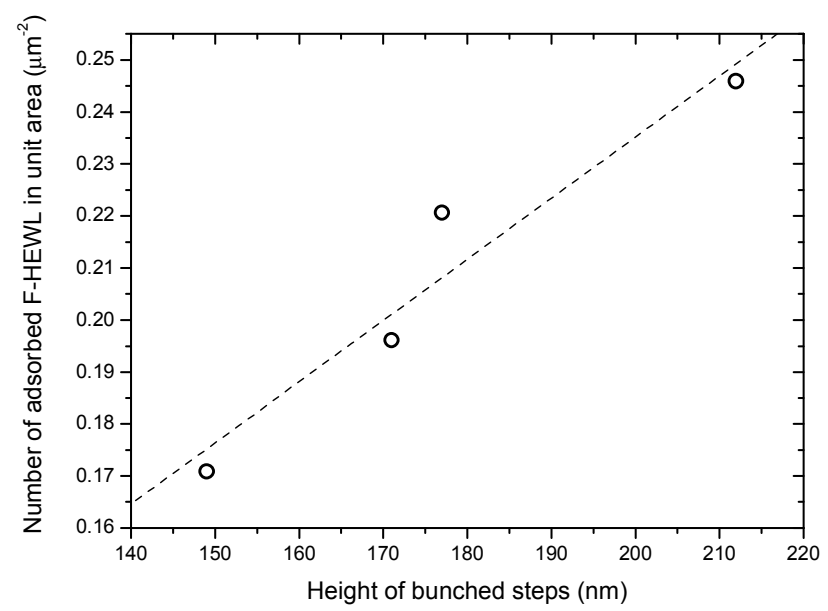

Figure 5. The number of adsorbed F-HEWL molecules on the bunched steps of HEWL crystal surface as a function of the height of bunched steps. The dashed line is the linear fit of the experimental data. The fitting equation is: $\mathrm{Y}=0.00118 \mathrm{X}, \mathrm{R}=0.96556$.

\section{REFERENCES}

[1] S. Weiss, "Fluorescence spectroscopy of single biomolecules," Science, vol. 283, pp. 1676-1682, 1999.

[2] H. P. Lu, "Probing single-molecule protein conformational dynamics," Accounts Chem. Res., vol. 38, pp. 557-565, 2005.

[3] X. Zhuang, and M. Rief, "Single-molecule folding," Curr. Opin. Struc. Biol., vol. 13, pp. 88-97, 2003.

[4] I. Rasnik, S. A. McKinney, and T. Ha, "Surfaces and Orientations: Much to FRET about?" Accounts Chem. Res., vol. 38, pp. 542-548, 2005.

[5] W. E. Moerner, "Optical measurements of single molecules in cells," TrAC Trends Anal. Chem., vol. 22, pp. 544-548, 2003.

[6] M., Cotlet, et al., "Probing conformational dynamics in single donoracceptor synthetic molecules by means of photoinduced reversible electron transfer," P. Natl. Acad. Sci. USA, vol. 101, pp. 14343-14348, 2004.

[7] T.-H. Lee, J. I. Gonzalez, J. Zheng, and R. M. Dickson, "SingleMolecule Optoelectronics," Accounts Chem. Res., vol. 38, pp. 534-541, 2005 .

[8] P. F. Barbara, A. J. Gesquiere, S.-J. Park, and Y. J. Lee, "SingleMolecule Spectroscopy of Conjugated Polymers," Accounts Chem. Res., vol. 38, pp. 602-610, 2005.

[9] T. Matsui, et al., "Impurity effects of lysozyme molecules specifically labeled with a fluorescent reagent on the crystallization of tetragonal and monoclinic lysozyme crystals," J. Cryst. Growth, vol. 293, pp. 415-422, 2006.

[10] G. Sazaki, et al., "In situ observation of elementary growth steps on the surface of protein crystals by laser confocal microscopy," J. Cryst. Growth, vol. 262, pp. 536-542, 2004.

[11] T. Ihle, C. Misbah, and O. Pierre-Louis, "Equilibrium step dynamics on vicinal surfaces revisited,” Phys. Rev. B, vol. 58, pp. 2289-2309, 1998.

[12] Y. Iimura, I. Yoshizaki, H. Nakamura, S. Yoda, and H. Komatsu, "Novel method for measuring the distribution coefficient in protein crystals," Cryst. Growth Des., vol. 5, pp. 301-305, 2005. 